\title{
HAUS8 regulates RLR-VISA antiviral signaling positively by targeting VISA
}

\author{
TIAN-SHENG HE ${ }^{1,2}$, TIAN CHEN ${ }^{1}$, DAN-DAN WANG ${ }^{1}$ and LIANG-GUO XU ${ }^{1}$ \\ ${ }^{1}$ Key Laboratory of Functional Small Organic Molecules, Ministry of Education and College of Life Science, \\ Jiangxi Normal University, Nanchang, Jiangxi 330022; ${ }^{2}$ State Key Laboratory of Medicinal Chemical Biology, \\ College of Life Science, Nankai University, Tianjin 300071, P.R. China
}

Received April 7, 2017; Accepted March 9, 2018

DOI: $10.3892 / \mathrm{mmr} .2018 .9171$

\begin{abstract}
Mitochondrial anti-viral signaling protein (VISA), additionally termed MAVS, IPS-1 and Cardif, is located at the outer membrane of mitochondria and is an essential adaptor in the Rig-like receptor (RLRs) signaling pathway. Upon viral infection, activated RLRs interact with VISA on mitochondria, forming a RLR-VISA platform, leading to the recruitment of different TRAF family members, including TRAF3, TRAF2 and TRAF6. This results in the phosphorylation and nuclear translocation of interferon regulatory factors 3 and 7 (IRF3/IRF7) by TANK binding kinase 1 (TBK1) and/or IKKe, as well as activation of NF- $\mathrm{KB}$, to induce type I interferons (IFNs) and pro-inflammatory cytokines. It remains to be elucidated how VISA functions as a scaffold for protein complex assembly in mitochondria to regulate RLR-VISA antiviral signaling. In the present study, it was demonstrated that HAUS augmin like complex subunit 8 (HAUS8) augments the RLR-VISA-dependent antiviral signaling pathway by targeting the VISA complex. Co-immunoprecipitation verified that HAUS8 was associated with VISA and the VISA signaling complex components retinoic acid-inducible gene I (RIG-I) and TBK1 when the RLR-VISA signaling pathway was activated. The data demonstrated that overexpression of HAUS8 significantly promoted the activity of the transcription factors NF- $\kappa B$, IRF3 and the IFN- $\beta$ promoter induced by Sendai virus-mediated RLR-VISA signaling. HAUS8 increased the polyubiquitination of VISA, RIG-I and TBK1. Knockdown of HAUS8 inhibited the activation of the transcription factors IRF-3, NF- $\mathrm{BB}$ and the IFN- $\beta$ promoter triggered by Sendai
\end{abstract}

Correspondence to: $\mathrm{Dr}$ Liang-Guo $\mathrm{Xu}$, Key Laboratory of Functional Small Organic Molecules, Ministry of Education and College of Life Science, Jiangxi Normal University, 99 Ziyang Road, Nanchang, Jiangxi 330022, P.R. China

E-mail: xul@jxnu.edu.cn

Key words: mitochondrial anti-viral signaling protein, Rig-like receptor-mitochondrial anti-viral signaling protein dependent signaling, HAUS augmin like complex subunit 8, nuclear factor- $\kappa \mathrm{B}$, interferon regulatory factor 3 , ubiquitination virus. Collectively, these results demonstrated that HAUS8 may function as a positive regulator of RLR-VISA dependent antiviral signaling by targeting the VISA complex, providing a novel regulatory mechanism of antiviral responses.

\section{Introduction}

Innate immunity is the first line of host defense against viral infection. Upon viral infection, pattern recognition receptors, including Toll-like receptors and RIG-I-like receptors (RLRs), recognize viral RNAs, which initiate a series of signaling cascades. RLRs consist of retinoic acid-inducible gene I (RIG-I) and melanoma differentiation-associated protein 5 (MDA-5), which are cytosolic helicases sensing viral RNA (1-5). RIG-I and MDA5 are structurally similar, containing two $\mathrm{N}$-terminal caspase activation and recruitment domain (CARD) domains, a central DEAD box helicase/ATPase domain with RNA helicase activity. Although RIG-I and MDA5 recognize different viral RNAs, VISA (also known as MAVS, IPS-1 and Cardif) is an essential adaptor located on the outer membrane of mitochondria shared by RIG-I and MDA5. VISA has an N-terminal CARD domain for interaction with RIG-I and MDA5, a C-terminal transmembrane domain that inserts VISA on the outer membrane of the mitochondria and a central proline-rich domain that interacts with various downstream signaling proteins to transduce antiviral signals (6-11). Once RLRs recognize viral RNA, activated RIG-I and MDA5 translocate to mitochondria and bind to VISA. Activation of VISA at the mitochondria acts as a platform for the recruitment of various signaling molecules, including tank-binding kinase 1 (TBK1),

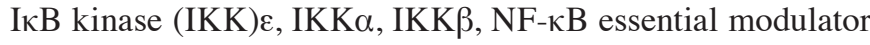
and other signaling proteins to form a VISA signalosome in the RLR-VISA signaling pathway. This results in the phosphorylation and activation of IFN regulatory factor (IRF)3/IRF7 by TBK1/IKKe and nuclear factor (NF)- $\kappa B$ through the IKK complex, inducing IFN and proinflammatory cytokine expression (8-13).

Although a number of interacting partners have been described in association with VISA (12), further investigation is required of the subtle regulatory mechanisms of VISA in antiviral signaling pathways through the identification of novel VISA interaction partners and its underlying signaling mechanisms. A yeast two-hybrid screen for VISA interacting 
partners identified HAUS augmin-like complex subunit 8 (HAUS8) as a VISA interacting partner. HAUS8 is a microtubule-associated protein essential for maintaining spindle integrity and chromosomal stability (14). The present study demonstrated that HAUS8 positively regulated the RLR-VISA dependent antiviral signaling pathway by recruiting the VISA complex, leading to promotion and activation of the transcription factors IRF3 and NF- $\mathrm{NB}$ to facilitate the activation of the IFN- $\beta$ promoter induced by Sendai virus infection. Further study additionally revealed that HAUS8 increased the polyubiquitination of VISA, RIG-I and TBK1. Collectively, HAUS8 may positively regulate the RLR-VISA antiviral signaling pathway.

\section{Materials and methods}

Cell culture and reagents. 293T cells were cultured in Dulbecco's modified Eagle's medium (Gibco; Thermo Fisher Scientific, Inc., Waltham, MA, USA) supplemented with $10 \%$ fetal bovine serum (Gibco; Thermo Fisher Scientific, Inc.) at $37^{\circ} \mathrm{C}$ in a $5 \% \mathrm{CO}_{2}$ incubator. Sendai virus was provided by $\mathrm{Dr}$ Hong-Bing Shu (Wuhan University, Wuhan, China). The mouse anti-Flag and anti-hemagglutinin (HA) monoclonal antibodies were obtained from Sigma-Aldrich; Merck KGaA (Darmstadt, Germany). The rabbit antibody against IRF3 (cat no. sc-9082) and the goat antibody against actin (cat no. sc-1616) were purchased from Santa Cruz Biotechnology, Inc. (Dallas, TX, USA). The dual-luciferase reporter assay system was obtained from Promega Corporation (Madison, WI, USA).

Constructs. CMV promoter-based mammalian expression plasmids for human Flag-tagged RIG-I, Flag-tagged N terminal of RIG-I (RIG-I-N; 1-284), Flag-tagged VISA, Flag-tagged TBK1, Flag-tagged TNF-receptor-associated factor (TRAF)3 and Flag-tagged IRF3-5D, HA-tagged ubiquitin, IFN- $\beta$ promoter luciferase reporter plasmid, ISRE luciferase reporter construct and $N F-\kappa B$ luciferase reporter construct $(8,15)$ were provided by Dr. Hong-Bing Shu. Human HAUS8 and mammalian expression vector pRK-5 (also provided by Dr. Hong-Bing Shu) with an N-terminal HA or Flag fusion tag were digested using the restriction enzymes Sal I and Not I (New England Biolabs, Inc., Ipswich, MA, USA), and constructed using ligase (New England Biolabs, Inc.) according to the manufacturer's instructions. An empty expression vector pRK-5 was used as a negative control in HAUS8 overexpression associated experiments. The human HAUS8 RNA interference (RNAi) constructs were generated using the pSuper. Retro vector (OligoEngine, Seattle, WA, USA), following protocols recommended by the manufacturer. A pSuper. Retro RNAi plasmid targeting green fluorescent protein (GFP) was used as a control in HAUS8 knockdown associated experiments. The target sequences for the human HAUS8 constructs were: small interfering (si)\#1, 5'-CCTGGATCTCTCTGCTATT-3'; si\#2, 5'-CCGGATTTATCTGAAGCAA-3'; si\#3, 5'-CCGTAA AGATGGAGAACAA-3'; and the target sequence used for GFP was 5'-GCAAGCTGACCCTGAAGTT-3' (provided by Dr. Hong-Bing Shu).

Yeast two-hybrid screen and dual-luciferase reporter assay. Yeast strain AH109, purchased from (Clontech Laboratories,
Inc., Mountainview, CA, USA), was transfected with plasmids containing $1 \mu \mathrm{g}$ human VISA (pGBT9-VISA; Clontech Laboratories, Inc.) and $100 \mu \mathrm{g}$ human 293T (pACT2; Clontech Laboratories, Inc.) using the lithium acetate/single-stranded carrier DNA/polyethylene glycol method (16). Positive clones were screened for via nutritional defects (Ser, Thr and His), and analyzed via sequencing (BGI, Shenzhen, China). 293T cells $\left(\sim 2.5 \times 10^{5}\right)$ were plated in 24 -well plates and transfected, using the calcium phosphate method (17), with plasmids carrying an IFN- $\beta$ promoter, ISRE or NF- $\kappa$ B luciferase reporter gene (firefly luciferase; $100 \mathrm{ng} /$ well) and pRL-TK (Renilla luciferase plasmid; $50 \mathrm{ng} /$ well) together with a doses of pRK5-HAUS8 $(0.05,0.1,0.2$ and $0.4 \mu \mathrm{g})$, GFP-siRNA or HAUS8-specific siRNAs $(0.5 \mu \mathrm{g})$, pRK5-RIG-I-N (1-284), pRK5-VISA, pRK5-TBK1 or pRK5-IRF3-5D [(in the presence or absence of HAUS8, (0.5 $\mu \mathrm{g}$ per plasmid)]. The same amount of total DNA in each transfection was normalized by added corresponding empty control plasmid or siGFP. A total of $12 \mathrm{~h}$ post-transfection, cells were infected with (+) or without (-) Sendai virus for $12 \mathrm{~h}$ in $37^{\circ} \mathrm{C}$ incubator as previously described $(1,8)$, at a multiplicity of infection of 5 plaque-forming units/cell, and cells were then collected and lysed using $1 \mathrm{X}$ passive lysis buffer included in the Dual-Luciferase ${ }^{\circledR}$ Reporter Assay System (cat. no. E1980; Promega Corporation). Subsequently, luciferase activity was measured with the dual-luciferase reporter assay system (Promega Corporation) with a GloMax ${ }^{\mathrm{TM}}$ 20/20 Luminometer (Promega Corporation) according to the manufacturer's protocols. Data was normalized by the ratio of firefly luciferase activity to Renilla luciferase activity, and the experiments were repeated three times.

Immunoprecipitation. $293 \mathrm{~T}$ cells $\left(\sim 6 \times 10^{6}\right)$ were seeded in $100 \mathrm{~mm}$ dishes and transfected with pRK5-Flag-RIG-I $(8 \mu \mathrm{g})$, pRK5-Flag-VISA $(8 \mu \mathrm{g})$, pRK5-Flag-TBK1 $(8 \mu \mathrm{g})$, pRK5-HA-HAUS8 $(8 \mu \mathrm{g})$, empty control plasmids $(8 \mu \mathrm{g})$ or pRK5-HA-ubiquitin $(8 \mu \mathrm{g}$; in the presence or absence of HAUS8) using the calcium phosphate method $(8,15)$. The same amount of total DNA in each transfection was normalized by added corresponding empty control plasmid. A total of $12 \mathrm{~h}$ post-transfection, cells were treated with or without Sendai virus for $12 \mathrm{~h}$ at $37^{\circ} \mathrm{C}$. A total of $22 \mathrm{~h}$ subsequent to transfection, cells were collected and lysed with lysis buffer containing $20 \mathrm{mM}$ Tris (pH 7.5), $150 \mathrm{mM} \mathrm{NaCl}, 1 \%$ Triton, 1 mM EDTA, $10 \mu \mathrm{g} / \mathrm{ml}$ aprotinin, $10 \mu \mathrm{g} / \mathrm{ml}$ leupeptin and $1 \mathrm{mM}$ phenylmethylsulfonyl fluoride (PMSF). For each immunoprecipitation, $0.5 \mathrm{ml}$ lysate was incubated with the appropriate amount of indicated antibody [anti-HA (1:1,500; cat. no. H3663; Sigma-Aldrich; Merck KGaA), anti-RIG-I, VISA, or TBK1 (1:1,000; cat. no. 8348; Cell Signaling Technology] and 30 $\mu 1$ protein G/A-Sepharose beads in $20 \%$ ethanol (GE Healthcare, Chicago, IL, USA) overnight at $4^{\circ} \mathrm{C}$. The Sepharose beads were washed three times with $1 \mathrm{ml}$ lysis buffer containing $500 \mathrm{mM} \mathrm{NaCl}$, and the precipitates were fractionated by $10 \%$ SDS/PAGE.

Western blot. Cells were lysed using the aforementioned lysis buffer for $1 \mathrm{~h}$ at $4^{\circ} \mathrm{C}$ as described above. Protein concentration was determined using a bicinchoninic acid protein assay kit (Tiangen Biotech Co., Ltd., Beijing, China). Proteins samples $(10 \mu \mathrm{g})$ were separated using $10 \%$ SDS-PAGE, transferred onto 
a nitrocellulose membrane (EMD Millipore, Billerica, MA, USA) and then blocked using 5\% non-fat milk for $1 \mathrm{~h}$ at room temperature. Membranes were then incubated with anti-Flag or anti-HA antibodies (1:4,000; cat. no. H3663; Sigma-Aldrich; Merck KGaA), anti-IRF3 (1:2,000; cat. no. sc-9082; Santa Cruz Biotechnology, Inc.), anti-P65 or anti-p-P65 (1:2,000; cat. no. 9936; Cell Signaling Technology, Inc.) and anti- $\beta$ actin (1:2,000; cat. no. sc-8432; Santa Cruz Biotechnology, Inc.) overnight at $4^{\circ} \mathrm{C}$. Membranes were then incubated with the following secondary antibodies for $1 \mathrm{~h}$ at $4^{\circ} \mathrm{C}$ : Horseradish peroxidase (HRP)-conjugated goat anti-mouse IgG Ab (1:5,000; cat. no. 1706516; Bio-Rad Laboratories, Inc., Hercules, CA, USA) or HRP-conjugated anti-rabbit IgG Ab (1:5,000; cat. no. 7074; Cell Signaling Technology, Inc.). Proteins were visualized using the enhanced chemiluminescent reagent in the Gel Doc XR Gel Documentation System (Bio-Rad Laboratories, Inc.) according to the manufacturer's instructions. The bands were analyzed using Image Lab software (version 5.1; Bio-Rad Laboratories, Inc.).

Reverse transcription-quantitative polymerase chain reaction $(R T-q P C R)$. Total RNA was extracted using an Eastep ${ }^{\circledR}$ Super total RNA Extraction Kit (Promega Corporation) according to the manufacturer's protocol, and cDNA was synthesized using an GoScript ${ }^{\mathrm{TM}}$ Reverse Transcription System (Promega Corporation). An Eastep ${ }^{\circledR}$ qPCR Master Mix kit (Promega Corporation) was used for real-time fluorescent qPCR assays. PCR thermocycling conditions were as follows: $50^{\circ} \mathrm{C}$ for $2 \mathrm{~min}$ and $95^{\circ} \mathrm{C}$ for $10 \mathrm{~min}$; followed by 40 cycles of denaturation at $95^{\circ} \mathrm{C}$ for $15 \mathrm{sec}$, annealing at $60^{\circ} \mathrm{C}$ for $1 \mathrm{~min}$; and a final extension at $72^{\circ} \mathrm{C}$ for $5 \mathrm{~min}$. Expression levels of genes of interest were normalized to the expression of $\beta$-actin. Relative gene expression data was analyzed using the $2^{-\Delta \Delta \mathrm{Cq}}$ method (18). The primers used for qPCR were as follows: Human (h)HAUS8 forward, 5'-AAGAGTTCAAGGTGGAAG AGTGA-3'; hHAUS8 reverse, 5'-TCAGACATCTTCCCTCGG GT-3'; hIFN- $\beta$ forward, 5'-CTAACTGCAACCTTTCGA AGC-3'; hIFN- $\beta$ reverse, 5 '-GGAAAGAGCTGTAGTGGA GAAG-3'; h $\beta$-actin forward, 5'-GTCGTCGACAACGGCTCC GGCATG-3'; and $h \beta$-actin reverse, 5'-ATTGTAGAAGGT GTGGTGCCAGAT-3'.

Native PAGE assay. 293T cells $\left(\sim 1 \times 10^{6}\right)$ were seeded in 6-well plates and transfected with the indicated plasmids (pRK5-Flag-HAUS8, empty vectors, GFP-RNAi or \#3 HAUS8 RNAi plasmids; $2.5 \mu \mathrm{g}$ each per well). A total of $12 \mathrm{~h}$ post-transfection, cells were infected with $(+)$ or without (-) Sendai virus for $12 \mathrm{~h}$ at $37^{\circ} \mathrm{C}$, and followed by collecting and lysing cells using native lysis buffer for $1 \mathrm{~h}$ at $4^{\circ} \mathrm{C}$, which was contained $50 \mathrm{mM}$ Tris ( $\mathrm{pH} 8.0$ ), $150 \mathrm{mM} \mathrm{NaCl}, 1 \%$ NP-40, $1 \mathrm{mM}$ EDTA, $1 \mathrm{mM}$ PMSF and $0.5 \%$ Sodium deoxycholate (19). The cell lysates in native PAGE sample buffer [62.5 mM Tris- $\mathrm{Cl}$ ( $\mathrm{pH}$ 6.8), 15\% glycerol and 1\% deoxycholate] were separated on a native PAGE and then immunoblotted with an anti-IRF3 antibody $(19,20)$.

Statistical analysis. All data presented in the histograms are presented as the mean \pm standard deviation of at least three independent experiments. The Student's t-test was performed to compare the statistical significance of differences between two groups, and one-way analysis of variance with Tukey's post hoc analysis was used for multiple comparisons $(n \geq 3)$. These statistical analyses were performed using GraphPad Prism software (version 5.0; GraphPad Software, Inc., La Jolla, CA, USA). P $<0.05$ was considered to indicate a statistically significant difference.

\section{Results}

Identification of HAUS8 as a positive regulator of virus-mediated RLR-VISA signaling. As VISA serves a key role in the antiviral signaling pathway, the present study attempted to further elucidate the function and underlying mechanism of VISA by looking for novel VISA interaction proteins. A systematic search for VISA interacting proteins in a yeast two-hybrid screen $(8,21)$ was performed, and HAUS8 was identified to be one of the positive clones by gene sequencing analysis (data not shown). Further co-immunoprecipitation and immunoblot analyses were performed to verify whether HAUS8 functions in the RLR-VISA signaling pathway. 293T human kidney cells were transfected with empty vector or HA-HAUS8 expression vector, and plasmids for Flag-tagged RIG-I, VISA and TBK. A total of $12 \mathrm{~h}$ post-transfection, the cells were infected with (+) or without (-) Sendai virus, followed by co-immunoprecipitation with anti-HA beads and western blot analysis with anti-Flag antibodies. The data revealed that HAUS8 interacted with RIG-I, VISA and TBK1. The association between HAUS8 and the three signaling molecules was augmented following infection with Sendai virus, and HAUS8 was obviously associated with RIG-I upon Sendai virus infection (Fig. 1A). These results suggested that HAUS8 may serve a role in the RLR-VISA signaling pathway by associating with the VISA signalosome.

To examine whether HAUS8 may be involved in the regulation of RLR-VISA mediated IFN induction, reporter assays were performed. The results showed that HAUS8 itself had no effect on activation of the IFN- $\beta$ promoter, ISRE and NF-кB. However, HAUS8 potentiated Sendai virus, a negative sense single-stranded RNA virus recognized by RIG-I (2), and induced activation of the IFN- $\beta$ promoter, ISRE and NF- $\kappa B$ in a dose-dependent manner (Fig. 1B). As activation of the IFN- $\beta$ promoter requires coordinated and cooperative activation of IRF3 and NF- $\mathrm{BB}(22-25)$, the present study further determined whether HAUS8 potentiated virus-induced activation of transcriptional factor IRF3 and NF- $\mathrm{kB}$. Consistently, overexpression of HAUS8 further increased the IRF3 dimerization and $\mathrm{P}-65$ phosphorylation caused by $\mathrm{SeV}$ infection (Fig. 1C and D). NF-kB activation was measured by P65 phosphorylation, and IRF3 activation was measured by its dimerization. These results suggested that HAUS8 potentiated virus-mediated RLR-VISA dependent IFN induction.

Knockdown of HAUS8 inhibits IFN- $\beta$ and antiviral responses. As overexpression of HAUS8 potentiated virus-mediated activation of NF- $\mathrm{kB}$ and IRF3, leading to activation of the IFN- $\beta$ promoter, it was next determined whether endogenous HAUS8 is required for virus-mediated IFN- $\beta$ induction by specific knockdown of HAUS8 endogenous expression.

To test this, the present study generated three siRNA expression RNAi plasmids (pSuper-HAUS8-RNAi\#1-3) that targeted HAUS8 mRNA, and their effects on the knockdown 

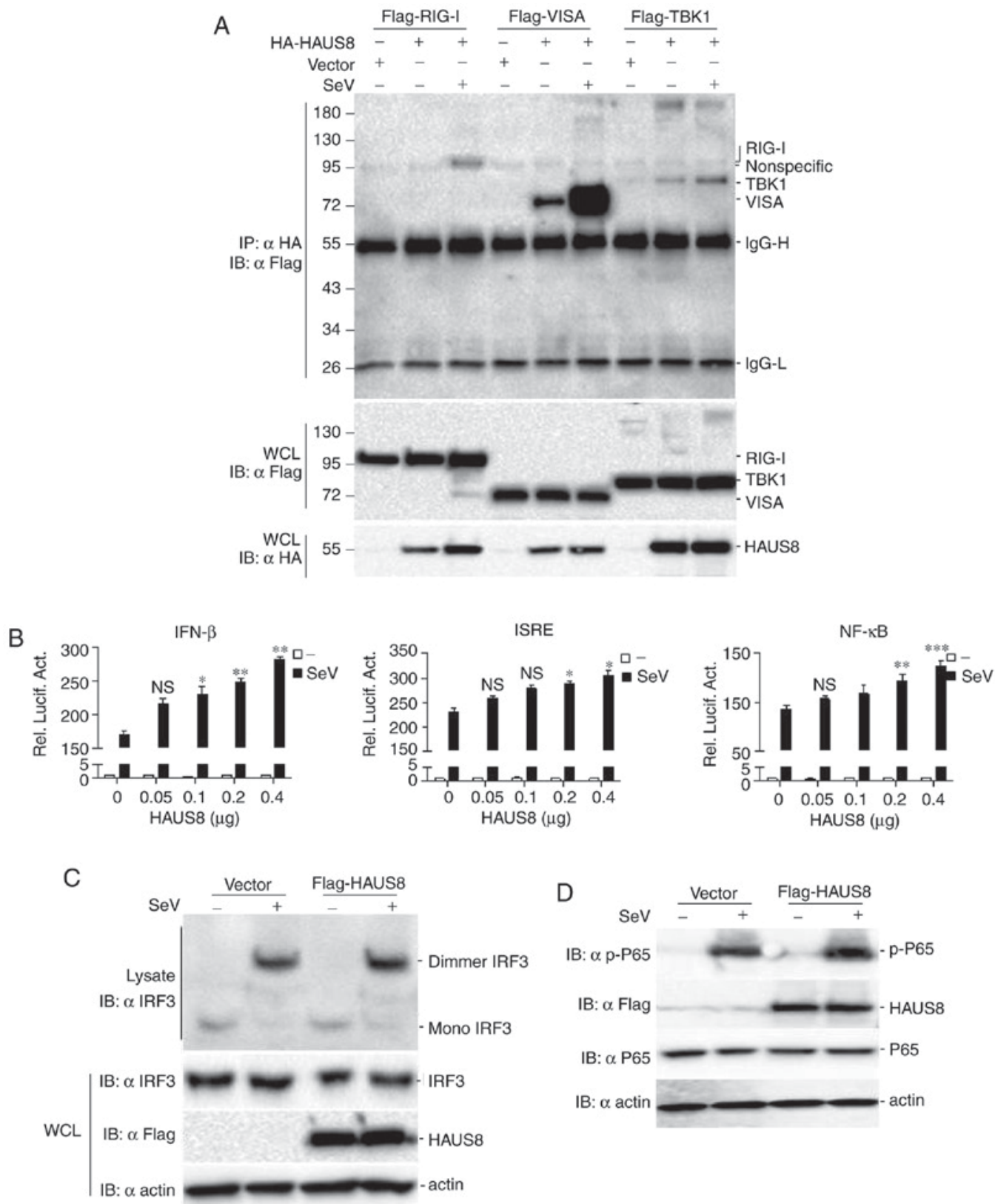

Figure 1. Identification of HAUS8 as a positive regulator of virus-mediated RLR-VISA signaling. (A) HAUS8 was associated with the RLR-VISA signaling complex. 293T human kidney cells $\left(\sim 6 \times 10^{6}\right)$ seeded in $100-\mathrm{mm}$ dishes were transfected with the indicated plasmids [pRK5-Flag-RIG-I, pRK5-Flag-VISA, pRK5-Flag-TBK1, pRK5-HA-HAUS8 or empty control plasmids $(8 \mu \mathrm{g}$ each)]. Following $12 \mathrm{~h}$ of transfection, cells were infected with or without Sendai virus for $10 \mathrm{~h}$ in $37^{\circ} \mathrm{C}$, then cells were harvested and lysed, and co-immunoprecipitation was performed with anti-HA. Immunoblot analysis was performed with anti-Flag antibodies (upper panels). Expression levels of the proteins were analyzed by immunoblot analysis of the lysates with anti-HA and anti-Flag antibodies (lower panels). (B) HAUS8 activates the IFN- $\beta$ promoter, ISRE and NF- $\mathrm{KB}$. 293T cells $\left(\sim 2.5 \times 10^{5}\right)$ seeded in 24 -well dishes were transfected with plasmids carrying an IFN- $\beta$ promoter, ISRE or NF- $\mathrm{BB}$ luciferase reporter gene (firefly luciferase; $100 \mathrm{ng} / \mathrm{well}$ ) and pRL-TK (Renilla luciferase plasmid; $50 \mathrm{ng} /$ well), together with an empty vector or the indicated dose of the HAUS8 plasmid $(0.05,0.1,0.2 \mathrm{and} 0.4 \mu \mathrm{g})$. Following $12 \mathrm{~h}$ of transfection, cells were infected with or without Sendai virus for $12 \mathrm{~h}$ at $37^{\circ} \mathrm{C}$. Cells were harvested for luciferase assay. Data were normalized by the ratio of firefly luciferase activity to Renilla luciferase activity. HAUS8 enhanced the activation of IRF3 and NF-kB induced by Sendai virus. 293T cells $\left(\sim 1 \times 10^{6}\right)$ seeded in 6 -well dishes were transfected with empty vector or HAUS8 plasmids ( $2.5 \mu \mathrm{g} /$ well). Following $12 \mathrm{~h}$ of transfection, cells were infected with (+) or without (-) Sendai virus for $12 \mathrm{~h}$ at $37^{\circ} \mathrm{C}$. Subsequently, cells were collected and lysed, and analyzed for (C) IRF3 dimerization by native gel electrophoresis or (D) P65 phosphorylation by SDS-PAGE. Error bars indicate standard deviations. ${ }^{*} \mathrm{P}<0.05 ;{ }^{* *} \mathrm{P}<0.01 ;{ }^{* * *} \mathrm{P}<0.001$; ns, no significant difference. HAUS8, HAUS augmin like complex subunit 8; RLR-VISA, RIG-I-like receptors-virus induced signaling adapter; HA, hemagglutinin; IFN- $\beta$, interferon- $\beta$; ISRE, interferon stimulated response

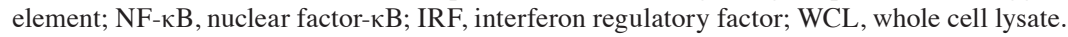

of HAUS8 expression were determined by western blotting (Fig. 2A, left) and RT-qPCR (Fig. 2A, right). The present study co-transfected a Flag-HAUS8 plasmid with an siRNA control or HAUS8-specific siRNAs into 293T cells, and transfected TRAF3 as the internal control in western blotting experiments. All three siRNAs partially inhibited the expression of transfected HAUS8 (Fig. 2A). To determine whether HAUS8 serves a role in the virus-mediated activation of NF- $\kappa \mathrm{B}$, IRF3 and the IFN- $\beta$ promoter, the HAUS8 RNAi vectors were transfected into 293T cells and reporter assays were performed. As presented in Fig. 2B, knockdown of HAUS8 expression inhibited Sendai virus-induced ISRE, NF- $\kappa$ B and IFN- $\beta$ promoter 

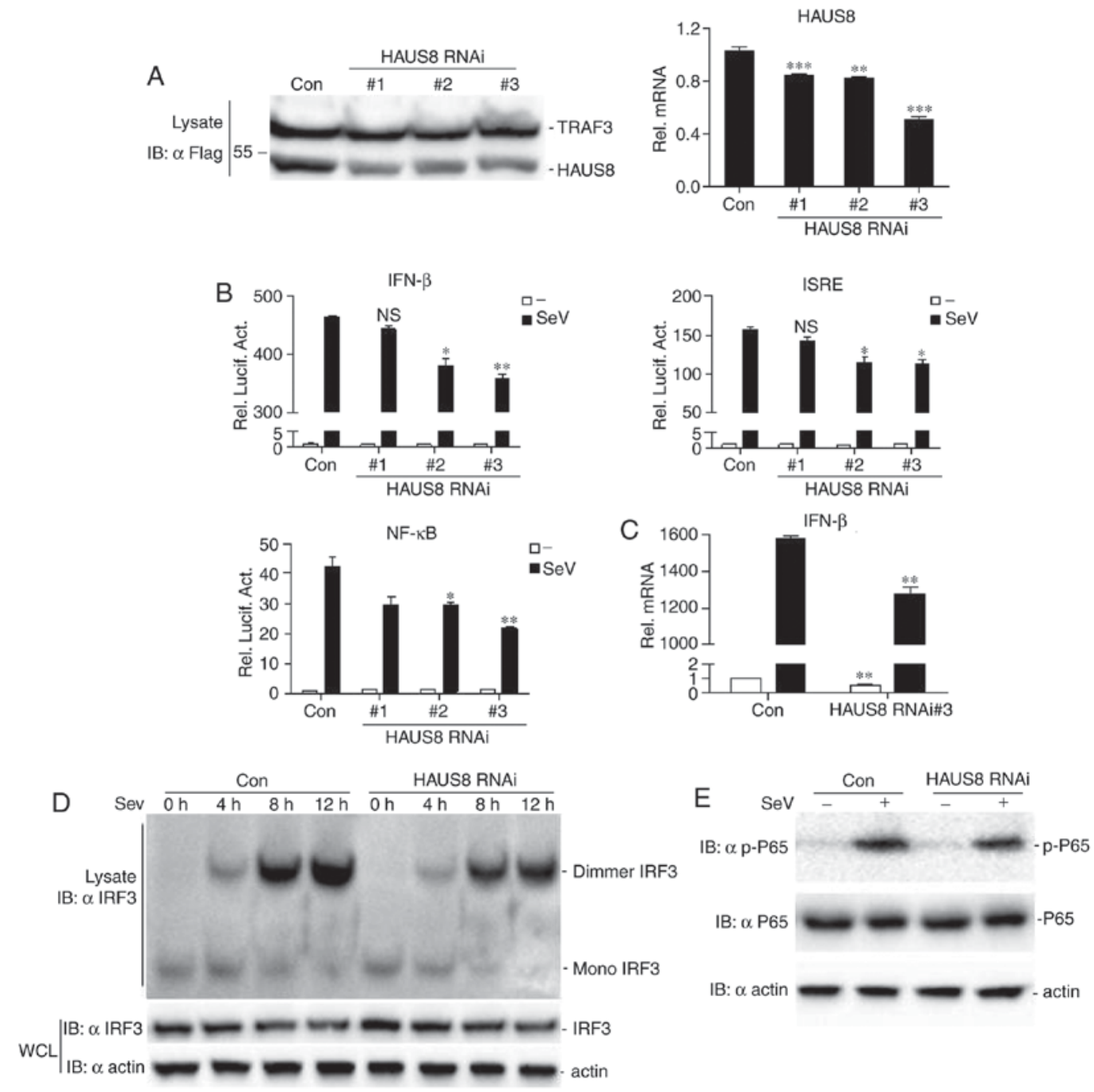

Figure 2. Knockdown of HAUS8 inhibits IFN- $\beta$ and antiviral responses. (A) Effects of HAUS8 RNAi constructs on the overexpression of HAUS8 by immunoblot analysis (left) or at the mRNA level by RT-qPCR (right). 293T cells $\left(\sim 1 \times 10^{6}\right)$ were seeded in 6 -well dishes and were transfected with Flag-HAUS8 $(1.5 \mu \mathrm{g})$, Flag-TRAF3 $(0.5 \mu \mathrm{g}$, as the internal control), together with HAUS8-specific siRNA or control RNAi vector $(2 \mu \mathrm{g})$. Following $24 \mathrm{~h}$ of transfection, cells were lysed, and the lysates were analyzed by western blotting with anti-Flag antibodies. 293T cells $\left(\sim 1 \times 10^{6}\right)$ seeded in 6-well dishes were transfected with HAUS8-specific siRNA or control RNAi vector $(3 \mu \mathrm{g})$. Following $24 \mathrm{~h}$ of transfection, cells were lysed for extracting RNA plasmids and RT-qPCR was performed. (B) Effect of HAUS8 RNAi constructs on Sendai virus-induced IFN- $\beta$, ISRE or NF-kB activation was determined. 293T cells $\left(\sim 2.5 \times 10^{5}\right)$ seeded in 24-well dishes were transfected with an IFN- $\beta$, ISRE or NF-кB luciferase plasmid (firefly luciferase; $100 \mathrm{ng} / \mathrm{well}$ ), pRL-TK (internal control Renilla luciferase plasmid; $50 \mathrm{ng} /$ well), together with HAUS8-RNAi plasmids or control RNAi vector $(0.5 \mu \mathrm{g})$. Following $12 \mathrm{~h}$ of transfection, cells were infected with or without Sendai virus for $12 \mathrm{~h}$ prior to the luciferase assays being performed. (C) RT-qPCR analysis of IFN- $\beta$ mRNA was performed. 293T cells $\left(\sim 1 \times 10^{6}\right)$ seeded in 6-well dishes were transfected with control RNAi or \#3 HAUS8 RNAi plasmids ( $3 \mu \mathrm{g})$, and $12 \mathrm{~h}$ subsequent to transfection, cells were treated with or without Sendai virus for $10 \mathrm{~h}$ prior to RT-qPCR being performed. Knockdown by HAUS8 RNAi inhibited the activation of IRF3 and NF-kB induced by Sendai virus. 293T cells $\left(\sim 1 \times 10^{6}\right)$ seeded in 6-well dishes were transfected with control RNAi or \#3 HAUS8 RNAi plasmids (2.5 $\mu \mathrm{g} /$ well). Following $12 \mathrm{~h}$ of transfection, the cells were infected with or without Sendai virus for the indicated time periods for (D) IRF3 activation detection, or $12 \mathrm{~h}$ for (E) NF- $\mathrm{kB}$ activation detection. Error bars indicate the standard deviation. ${ }^{*} \mathrm{P}<0.05 ;{ }^{* *} \mathrm{P}<0.01 ;{ }^{* * *} \mathrm{P}<0.001 ;$ ns, no significant difference; RT-qPCR, reverse transcription-quantitative polymerase chain reaction; HAUS8, HAUS augmin like complex subunit 8; IFN- $\beta$, interferon- $\beta$; RNAi, RNA interference; siRNA, small interfering RNA; ISRE, interferon stimulated response element; NF- $\mathrm{kB}$, nuclear factor- $\mathrm{kB}$; SeV, Sendai virus; IRF, interferon regulatory factor; WCL, whole cell lysate; con, control; TRAF, tumor necrosis factor receptor-associated factor.

activation. The degree of inhibition was associated with the efficiency of knockdown of HAUS8 expression by each RNAi vector. The HAUS8-RNAi-\#3 construct was selected for all additional experiments described below as it demonstrated the greatest knockdown of HAUS8. Transfection with HAUS8-RNAi-\#3 resulted in lower virus-mediated IFN- $\beta$ production at the mRNA level by RT-qPCR analysis (Fig. 2C).

Furthermore, control RNAi or HAUS8 RNAi were transfected into 293T cells, then the cells were infected with (+) or without (-) Sendai virus for different time periods to determine
IRF3 activation by measuring IRF3 dimerization. As presented in Fig. 2D, HAUS8 knockdown decreased the dimerization of endogenous IRF3 at different time points. Knockdown of HAUS8 decreased P65 phosphorylation induced by Sendai virus (Fig. 2E). Together, these data suggested that HAUS8 may be required for virus-mediated IRF3 or P65 activation and IFN- $\beta$ promoter activation.

HAUS8 regulates antiviral signaling via RLR-VISA dependent signaling downstream of VISA signalosome. The effect of 

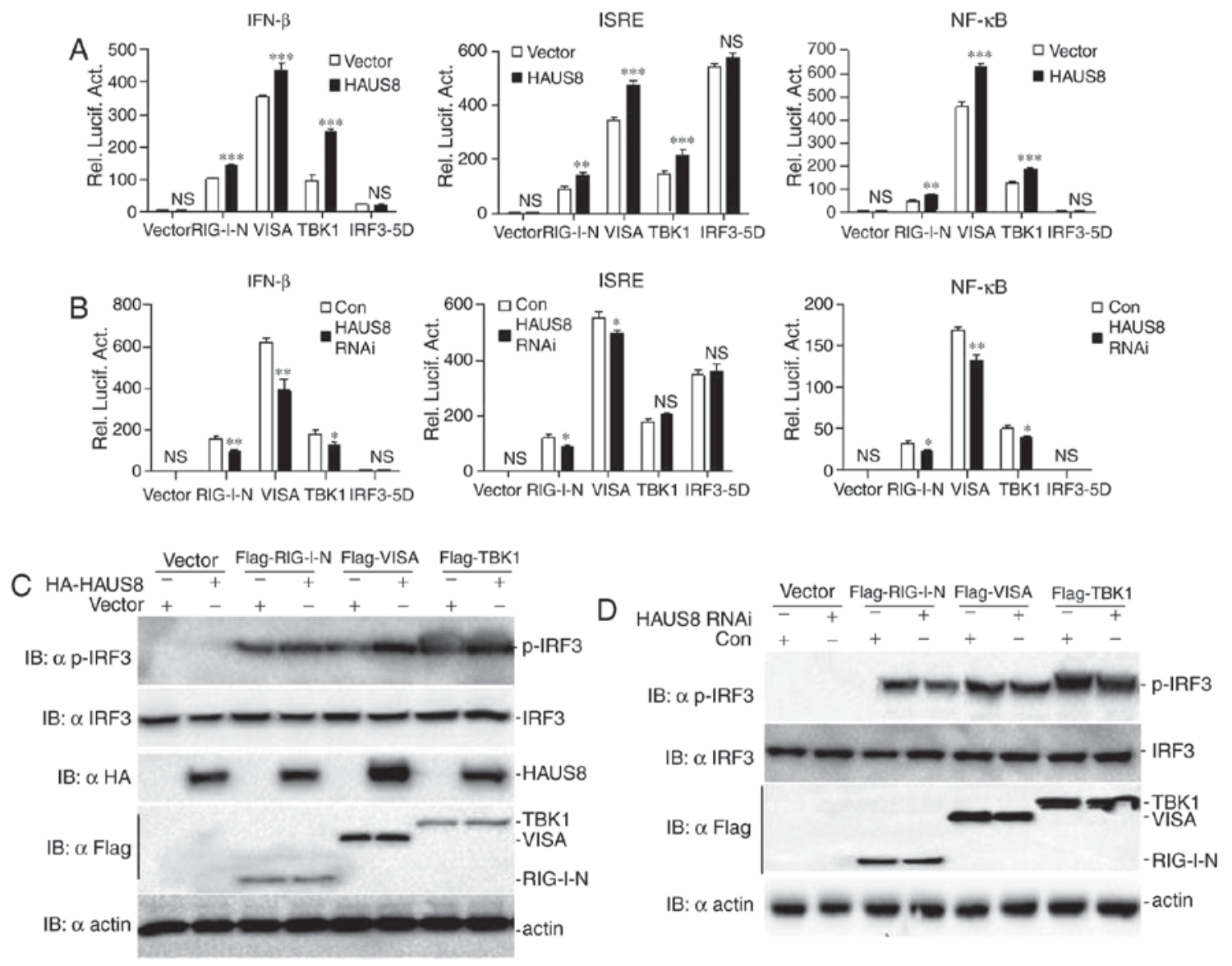

Figure 3. HAUS8 regulates antiviral signaling via RLR-VISA dependent signaling downstream of the VISA signalosome. (A) HAUS8 augmented RIG-I-, VISA- and TBK1-mediated activation of IFN- $\beta$ promoter, ISRE or NF- $\mathrm{\kappa B}$. 293T cells $\left(\sim 2.5 \times 10^{5}\right)$ seeded in 24 -well dishes were transfected with plasmids carrying an IFN- $\beta$ promoter, ISRE or NF- $\mathrm{kB}$ luciferase reporter gene (firefly luciferase; $100 \mathrm{ng} / \mathrm{well}$ ) and pRL-TK (Renilla luciferase plasmid; $50 \mathrm{ng} / \mathrm{well}$ ), together with empty vector or HAUS8 expression vector $(0.5 \mu \mathrm{g})$, and the indicated expression vector [pRK5-RIG-I-N (1-284), pRK5-VISA, pRK5-TBK1 or pRK5-IRF3-5D (0.5 $\mu \mathrm{g}$ each)]. Luciferase assays were performed $24 \mathrm{~h}$ after transfection. (B) HAUS8 knockdown inhibited RIG-I-, VISA- and TBK1-mediated activation of IFN- $\beta$ promoter, ISRE or NF- $\mathrm{kB}$. 293T cells $\left(\sim 2.5 \times 10^{5}\right)$ seeded in 24 -well dishes were transfected with plasmids carrying an IFN- $\beta$ promoter, ISRE or NF-kB luciferase reporter gene (firefly luciferase; $100 \mathrm{ng} /$ well) and pRL-TK (Renilla luciferase plasmid; $50 \mathrm{ng} /$ well), together with RNAi control or HAUS8 RNAi $(0.5 \mu \mathrm{g})$, and the indicated expression vector [pRK5-RIG-I-N (1-284), pRK5-VISA, pRK5-TBK1 or pRK5-IRF3-5D (0.5 $\mu \mathrm{g}$ each]. Luciferase assays were performed $24 \mathrm{~h}$ post-transfection. (C) HAUS8 positively regulated RIG-I-, VISA- and TBK1-mediated activation of phosphorylated IRF3 and TBK1. 293T cells $\left(\sim 1 \times 10^{6}\right)$ seeded in 6-well dishes were transfected with empty vector or HAUS8 expression vector $(3 \mu \mathrm{g})$, together with the indicated expression vector $(2 \mu \mathrm{g})$. Following $24 \mathrm{~h}$ of transfection, cells were harvested for detecting the phosphorylation of IRF3 and TBK1. (D) HAUS8 knockdown inhibited RIG-I-, VISA- and TBK1-mediated activation of phosphorylation IRF3 and TBK1. 293T cells $\left(\sim 1 \times 10^{6}\right)$ seeded in 6-well dishes were transfected with control RNAi or HAUS8 RNAi $(3 \mu \mathrm{g})$, together with the indicated expression vector $(2 \mu \mathrm{g})$. Following $24 \mathrm{~h}$ of transfection, cells were harvested for detecting the phosphorylated IRF3 and TBK1. Error bars indicated standard deviation. ${ }^{*} \mathrm{P}<0.05 ;{ }^{* *} \mathrm{P}<0.01 ;{ }^{* * *} \mathrm{P}<0.001 ;$ ns, no significant difference. HAUS8, HAUS augmin like complex subunit 8; RLR-VISA, RIG-I-like receptors-virus induced signaling adapter; HA, hemagglutinin; IFN- $\beta$, interferon- $\beta$; TBK1, TANK-binding

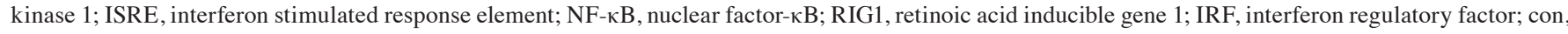
control; rel. lucif. act., relative luciferase activity.

HAUS8 on RLR-VISA-induced IFN signaling was investigated to determine whether HAUS8 acts upstream or downstream of the VISA signalosome. In the luciferase reporter assay, it was demonstrated that HAUS8 promoted VISA-, RIG-I- and TBK1-mediated activation of ISRE, NF- $\kappa B$ and the IFN- $\beta$ promoter (Fig. 3A).

In addition, the assay was performed to detect the effect of HAUS8 knockdown on RLR-VISA dependent signaling. It was found that HAUS8 knockdown markedly inhibited RIG-I-, VISA- and TBK1-, although not IRF3-mediated activation of ISRE, NF- $\kappa \mathrm{B}$ and IFN- $\beta$ promoter (Fig. 3B).

It was also demonstrated that HAUS8 increased VISA-, RIG-I- and TBK1-mediated IRF3 activation (Fig. 3C), and HAUS8 knockdown decreased RIG-I-, VISA- and TBK1-mediated IRF3 activation (Fig. 3D). Taken together, these data suggested that HAUS8 may act as an active partner to regulate virus-mediated RLR-VISA signaling by targeting downstream of the VISA signalosome.

HAUS8 enhances the polyubiquitination of VISA, RIG-I and TBK1. Tripartite motif-containing (TRIM) 25 is a ubiquitin E3 ligase that serves a key role in virus-induced RIG-I activation, by mediating the ubiquitination and oligomerization of RIG-1 and its interaction with VISA (26). A number of other E3 ligases, including ring finger protein 135 (27), TRIM4 (28) and mex-3 RNA binding family member C (29) have additionally been identified as important regulators in virus-induced IFN induction by promoting K63-linked ubiquitination for RIG-I activation.

The post-translational modification and ubiquitination of VISA, and its interacting partners, is a key aspect of host cell 

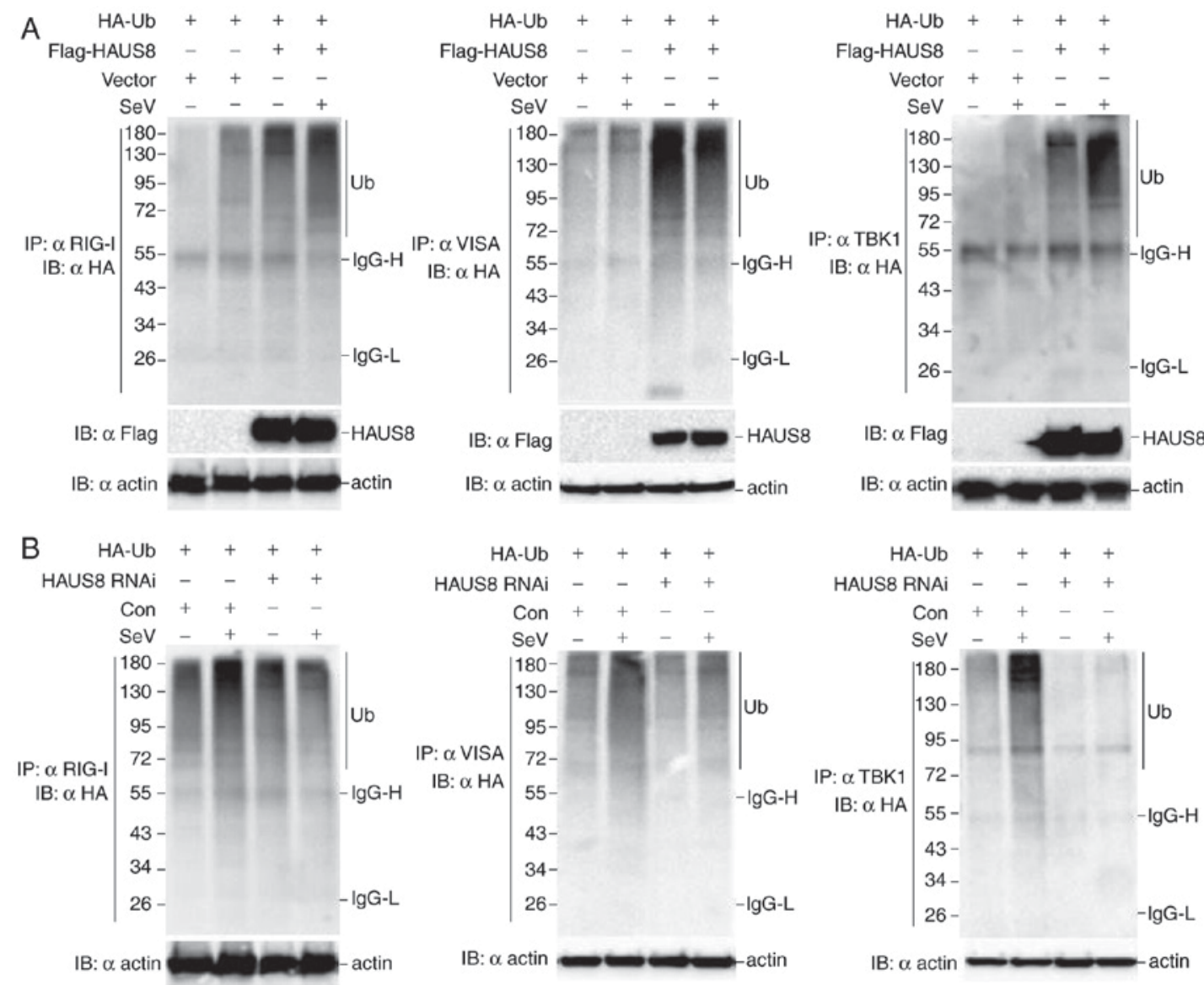

Figure 4. HAUS8 enhances the polyubiquitination of VISA, RIG-I and TBK1. (A) 293T cells $\left(\sim 6 \times 10^{6}\right)$ seeded in 100 -mm dishes were transfected with empty vector or Flag-HAUS $8(8 \mu \mathrm{g})$, and HA-ubiquitin $(8 \mu \mathrm{g})$. Following $12 \mathrm{~h}$ of transfection, cells were infected with or without Sendai virus for $10 \mathrm{~h}$; immunoprecipitation was performed using anti-RIG-I, anti-VISA or anti-TBK1 beads and immunoblotting analysis were performed with anti-HA antibodies. (B) 293T cells $\left(\sim 6 \times 10^{6}\right)$ seeded in 100-mm dishes were transfected with an RNAi control or HAUS8 RNAi $(8 \mu \mathrm{g})$ and HA-ubiquitin $(8 \mu \mathrm{g})$. Following $12 \mathrm{~h}$ of transfection, cells were infected with or without Sendai virus for $10 \mathrm{~h}$; immunoprecipitation was performed with anti-RIG-I, anti-VISA or anti-TBK1 beads and immunoblotting analysis was performed with anti-HA antibodies. HAUS8, HAUS augmin like complex subunit 8; VISA, virus induced signaling adapter; HA, hemagglutinin; TBK1, TANK-binding kinase 1; RIG1, retinoic acid inducible gene 1; RNAi, RNA interference; Ub, ubiquitin; SeV, Sendai virus.

regulation of antiviral signaling. Nedd4 family interacting protein 1 (30), proteasome subunit a type 7 (31), TRIM25 (32) and other proteins (12) have been reported to enhance E3 ligase-mediated VISA degradation, leading to negative or positive regulation of RLR-VISA dependent immune signaling.

It has been reported that the $\mathrm{E} 3$ ubiquitin ligases ring finger protein 128 (33), mindbomb E3 ubiquitin protein ligase (MIB)1, MIB2 (34) and E3 ubiquitin ligase Nrdp1 (35) positively activate TBK1 by promoting its K63-linked polyubiquitination during viral infection, and other proteins such as NLR family pyrin domain containing 4 (36) and TRAF-interacting protein (37) was reported to negatively regulate type I IFN signaling by targeting the kinase TBK1.

To determine whether HAUS8 regulates the ubiquitination of VISA, RIG-I and TBK1, 293T cells were transfected with the Flag-HAUS8 or empty vector as a control and HA-tagged ubiquitin, and subsequently infected with or without Sendai virus. A coimmunoprecipitation assay was performed using anti-VISA, anti-RIG-I and anti TBK1 as immunoprecipitation antibodies. The results revealed that HAUS8 markedly augmented the ubiquitination of VISA, RIG-I and TBK1, and this ubiquitination increased more following Sendai virus infection (Fig. 4A). Consistent with these results, HAUS8 knockdown attenuated the ubiquitination of VISA, RIG-I and TBK1 under Sendai virus infection (Fig. 4B). The results suggested that HAUS8 positively regulates RLR-mediated antiviral signaling by promoting the polyubiquitination of VISA, RIG-I and TBK1 when RLR-VISA signaling is activated.

\section{Discussion}

VISA serves vital roles in RLR signaling by recruiting activated RIG-I and MDA5 and downstream signaling proteins to form a protein complex assembly as a platform. Although multiple partners for the VISA signalosome were identified to regulate its function by diverse mechanisms, including the spatiotemporal distribution, mitochondrial dynamics and post-translational modifications, including phosphorylation and ubiquitination $(13,38)$, there remains a lack of comprehensive understanding of the function of VISA. The present study attempted to investigate how VISA fine-tunes RLR-mediated antiviral signaling.

It has been reported that the cytoskeletal system, including microtubules and actin filaments, serves an important role in antiviral signaling $(39,40)$. Focal adhesion kinase (FAK) is a protein tyrosine kinase that is located at focal 
adhesions to connect the intracellular cytoskeleton and the extracellular matrix. FAK interacts with VISA in a viral infection-dependent manner and potentiates VISA-mediated signaling; virus-induced $\mathrm{NF}-\kappa \mathrm{B}$ and IFN- $\beta$ signaling in FAK-deficient mouse embryonic fibroblasts was attenuated in a previous study, and the overexpression of FAK in 293T cells enhanced the activation of the NF- $\mathrm{B}$ and IFN- $\beta$ promoters in response to Sendai virus infection (41). Rho guanine nucleotide exchange factor 2 (GEF-H1) is a microtubule-associated guanine nucleotide exchange factor for Rac and Rho GTPases. Overexpression of GEF-H1 in 293T cells enhanced VISA-mediated IRF3 phosphorylation and IFN- $\beta$ promoter activation, and GEF-H1-deficient macrophages exhibited significantly less IRF3 phosphorylation and IFN- $\beta$ promoter activation in response to VISA expression compared with wild-type macrophages (42). GEF-H1-deficient macrophages are markedly defective in the induction of IFN- $\beta$ upon viral infection or under treatment with synthetic double stranded RNAs. Disruption of microtubule polarization inhibited the activation of GEF-H1 and, consequently, RLR signaling (42). It was additionally reported that actin was translocated onto the mitochondria, with faster kinetics compared with RIG-I, upon viral infection (43). Collectively, these previously published data indicate that, the host cytoskeletal system have involved in the activation of the NF- $\kappa \mathrm{B}$ and IFN- $\beta$ in innate immunity.

Consistent with previous findings $(14,39,40)$, a novel cytoskeleton protein HAUS8 was revealed to be involved in the innate immune response against RNA viruses in the cytoplasm in the present study. HAUS8 is a microtubule-associated protein and serves a notable role in maintaining spindle integrity and chromosome stability (14). In the present study, it was demonstrated that HAUS8 is recruited into the VISA complex by associating with the RIG-I/VISA/TBK1 components of the RLR-VISA signaling pathway, and overexpression of HAUS8 augmented the activation of the transcription factors IRF3, NF- $\kappa$ B and the IFN- $\beta$ promoter induced by Sendai virus-mediated RLR-VISA dependent antiviral signaling. Further investigation demonstrated that HAUS8 upregulates the ubiquitination of VISA, RIG-I and TBK1. The data suggested that HAUS8 acts as a positive regulator of RLR-VISA signaling.

The data of the present study demonstrated that HAUS8 associated with RIG-I only under viral infection, and the association between HAUS8 and VISA or TBK1 increased upon virus infection. The temporal and spatial associations between HAUS8 and RIG-I, VISA or TBK1 indicated that HAUS8 may transfer from the cytoplasm to the mitochondria and become a component of the RLR-VISA signaling platform to serve an important role in the redistribution of RIG-I or VISA.

HAUS8 knockdown may inhibit the ability of HAUS8 to redistribute RIG-I and VISA, leading to impaired activation of IRF3, NF- $\kappa$ B and the IFN- $\beta$ promoter induced by Sendai virus. The results suggested that HAUS8 may be required for the redistribution of RIG-I and VISA in RLR-VISA signaling.

In conclusion, the present study demonstrated a novel role of HAUS8, one of the subunits of augmin complex associated with microtubule generation, in the regulation of the RLR-VISA dependent signaling pathway, and may connect the cytoskeleton and RLR-VISA antiviral signaling. Given that HAUS8 is not an enzyme of the ubiquitination pathway, it was hypothesized that HAUS8 may be acting as a physical scaffold and may recruit certain ubiquitin-associated proteins to the VISA signalosome, promoting the polyubiquitination of VISA, RIG-I and TBK1, leading to activation of RLR-VISA signaling. Screening candidate E3 ubiquitin ligases that may work together with HAUS8 may help elucidate a novel mechanism of RLR-VISA regulation, and this requires further investigation.

\section{Acknowledgements}

The authors of the present study would like to thank Dr Hong-Bing Shu (Medical Research Institute, Wuhan University, Wuhan, China) for providing plasmids and other reagents.

\section{Funding}

This work was supported by grants from the National Natural Science Foundation of China (grant nos. 31370876 and 31570876), the Natural Science Foundation of Jiangxi Province (grant nos. 20143ACB20004 and 20161BAB204177), the Open Project Program of Key Laboratory of Functional Small Organic Molecule, Ministry of Education, and Jiangxi Normal University (grant no. KLFS-KF-201407) and the Graduate Innovation Fund of Jiangxi Normal University (grant no. YC2015-S141).

\section{Availability of data and materials}

All data generated or analyzed during this study are included in this published article.

\section{Authors' contributions}

LX conceived the study. LX and TH performed the analysis and wrote the manuscript. TH, TC and DW performed the assays. All authors read and approved the manuscript.

\section{Ethics approval and consent to participate}

Not applicable.

\section{Consent for publication}

Not applicable.

\section{Competing interests}

The authors declare that they have no competing interests.

\section{References}

1. Yoneyama M, Kikuchi M, Natsukawa T, Shinobu N, Imaizumi T, Miyagishi M, Taira K, Akira S and Fujita T: The RNA helicase RIG-I has an essential function in double-stranded RNA-induced innate antiviral responses. Nat Immunol 5: 730-737, 2004.

2. Kato H, Sato S, Yoneyama M, Yamamoto M, Uematsu S, Matsui K, Tsujimura T, Takeda K, Fujita T, Takeuchi O and Akira S: Cell type-specific involvement of RIG-I in antiviral response. Immunity 23: 19-28, 2005. 
3. Kato H, Takeuchi O, Sato S, Yoneyama M, Yamamoto M, Matsui K, Uematsu S, Jung A, Kawai T, Ishii KJ, et al: Differential roles of MDA5 and RIG-I helicases in the recognition of RNA viruses. Nature 441: 101-105, 2006.

4. Pichlmair A, Schulz O, Tan CP, Näslund TI, Liljeström P, Weber F and Reis e Sousa C: RIG-I-mediated antiviral responses to single-stranded RNA bearing 5'-phosphates. Science 314 997-1001, 2006.

5. Hornung V,Ellegast J,Kim S, BrzózkaK, Jung A, Kato H, Poeck H, Akira S, Conzelmann KK, Schlee M, et al: 5'-Triphosphate RNA is the ligand for RIG-I. Science 314: 994-997, 2006.

6. Takeuchi $\mathrm{O}$ and Akira S: Pattern recognition receptors and inflammation. Cell 140: 805-820, 2010.

7. Goubau D, Deddouche S and Reis e Sousa C: Cytosolic sensing of viruses. Immunity 38: 855-869, 2013.

8. Xu LG, Wang YY, Han KJ, Li LY, Zhai Z and Shu HB: VISA is an adapter protein required for virus-triggered IFN-beta signaling. Mol Cell 19: 727-740, 2005.

9. Seth RB, Sun L, Ea CK and Chen ZJ: Identification and characterization of MAVS, a mitochondrial antiviral signaling protein that activates NF-kappaB and IRF 3. Cell 122: 669-682, 2005.

10. Meylan E, Curran J, Hofmann K, Moradpour D, Binder M, Bartenschlager R and Tschopp J: Cardif is an adaptor protein in the RIG-I antiviral pathway and is targeted by hepatitis $\mathrm{C}$ virus. Nature 437: 1167-1172, 2005.

11. Kawai T, Takahashi K, Sato S, Coban C, Kumar H, Kato H, Ishii KJ, Takeuchi O and Akira S: IPS-1, an adaptor triggering RIG-I- and Mda5-mediated type I interferon induction. Nat Immunol 6: 981-988, 2005.

12. Jacobs JL and Coyne CB: Mechanisms of MAVS regulation at the mitochondrial membrane. J Mol Biol 425: 5009-5019, 2013.

13. Belgnaoui SM, Paz S and Hiscott J: Orchestrating the interferon antiviral response through the mitochondrial antiviral signaling (MAVS) adapter. Curr Opin Immunol 23: 564-572, 2011.

14. Wu G, Lin YT, Wei R, Chen Y, Shan Z and Lee WH: Hice1, a novel microtubule-associated protein required for maintenance of spindle integrity and chromosomal stability in human cells Mol Cell Biol 28: 3652-3662, 2008.

15. Zhong B, Yang Y, Li S, Wang YY, Li Y, Diao F, Lei C, He X, Zhang L, Tien P and Shu HB: The adaptor protein MITA links virus-sensing receptors to IRF3 transcription factor activation. Immunity 29: 538-550, 2008.

16. Gietz RD, Schiestl RH, Willems AR and Woods RA: Studies on the transformation of intact yeast cells by the LiAc/SS-DNA/PEG procedure. Yeast 11: 355-360, 1995.

17. Chenuet S, Martinet D, Besuchet-Schmutz N, Wicht M Jaccard N, Bon AC, Derouazi M, Hacker DL, Beckmann JS and Wurm FM: Calcium phosphate transfection generates mammalian recombinant cell lines with higher specific productivity than polyfection. Biotechnol Bioeng 101: 937-945, 2008.

18. Livak KJ and Schmittgen TD: Analysis of relative gene expression data using real-time quantitative PCR and the 2(-Delta Delta C(T)) method. Methods 25: 402-408, 2001.

19. Robitaille AC, Mariani MK, Fortin A and Grandvaux N: A high resolution method to monitor phosphorylation-dependent activation of IRF3. J Vis Exp: e53723, 2016.

20. Yamamoto M, Sato S, Hemmi H, Hoshino K, Kaisho T, Sanjo H, Takeuchi O, Sugiyama M, Okabe M, Takeda K and Akira S: Role of adaptor TRIF in the MyD88-independent toll-like receptor signaling pathway. Science 301: 640-643, 2003.

21. Fields S and Song O: A novel genetic system to detect protein-protein interactions. Nature 340: 245-246, 1989.

22. Maniatis T, Falvo JV, Kim TH, Kim TK, Lin CH, Parekh BS and Wathelet MG: Structure and function of the interferon-beta enhanceosome. Cold Spring Harb Symp Quant Biol 63: 609-620, 1998.

23. Silverman $N$ and Maniatis T: NF-kappaB signaling pathways in mammalian and insect innate immunity. Genes Dev 15 2321-2342, 2001

24. Hiscott J, Grandvaux N, Sharma S, Tenoever BR, Servant MJ and Lin R: Convergence of the NF-kappaB and interferon signaling pathways in the regulation of antiviral defense and apoptosis. Ann N Y Acad Sci 1010: 237-248, 2003.
25. Yoneyama M, Suhara W and Fujita T: Control of IRF-3 activation by phosphorylation. J Interferon Cytokine Res 22: 73-76, 2002.

26. Gack MU, Shin YC, Joo CH, Urano T, Liang C, Sun L, Takeuchi O, Akira S, Chen Z, Inoue S and Jung JU: TRIM25 RING-finger E3 ubiquitin ligase is essential for RIG-I-mediated antiviral activity. Nature 446: 916-920, 2007.

27. Oshiumi $H$, Matsumoto $M$, Hatakeyama $S$ and Seya $T$ : Riplet/RNF135, a RING finger protein, ubiquitinates RIG-I to promote interferon-beta induction during the early phase of viral infection. J Biol Chem 284: 807-817, 2009.

28. Yan J, Li Q, Mao AP, Hu MM and Shu HB: TRIM4 modulates type I interferon induction and cellular antiviral response by targeting RIG-I for K63-linked ubiquitination. J Mol Cell Biol 6: 154-163, 2014.

29. Kuniyoshi K, Takeuchi O, Pandey S, Satoh T, Iwasaki H, Akira S and Kawai T: Pivotal role of RNA-binding E3 ubiquitin ligase MEX3C in RIG-I-mediated antiviral innate immunity. Proc Natl Acad Sci USA 111: 5646-5651, 2014

30. Wang $\mathrm{Y}$, Tong $\mathrm{X}$ and Ye $\mathrm{X}$ : Ndfip1 negatively regulates RIG-I-dependent immune signaling by enhancing E3 ligase Smurf1-mediated MAVS degradation. J Immunol 189: 5304-5313, 2012.

31. Jia Y, Song T, Wei C, Ni C, Zheng Z, Xu Q, Ma H, Li L, Zhang Y, $\mathrm{He} X$, et al: Negative regulation of MAVS-mediated innate immune response by PSMA7. J Immunol 183: 4241-4248, 2009.

32. Castanier C, Zemirli N, Portier A, Garcin D, Bidère N, Vazquez A and Arnoult D: MAVS ubiquitination by the E3 ligase TRIM25 and degradation by the proteasome is involved in type I interferon production after activation of the antiviral RIG-I-like receptors. BMC Biol 10: 44, 2012.

33. Song G, Liu B, Li Z, Wu H, Wang P, Zhao K, Jiang G, Zhang L and Gao C: E3 ubiquitin ligase RNF128 promotes innate antiviral immunity through K63-linked ubiquitination of TBK1. Nat Immunol 17: 1342-1351, 2016.

34. Li S, Wang L, Berman M, Kong YY and Dorf ME: Mapping a dynamic innate immunity protein interaction network regulating type I interferon production. Immunity 35: 426-440, 2011.

35. Wang C, Chen T, Zhang J, Yang M, Li N, Xu X and Cao X: The E3 ubiquitin ligase Nrdp1 'preferentially' promotes TLR-mediated production of type I interferon. Nat Immunol 10: 744-752, 2009

36. Cui J, Li Y, Zhu L, Liu D, Songyang Z, Wang HY and Wang RF: NLRP4 negatively regulates type I interferon signaling by targeting the kinase TBK1 for degradation via the ubiquitin ligase DTX4. Nat Immunol 13: 387-395, 2012.

37. Zhang M, Wang L, Zhao X, Zhao K, Meng H, Zhao W and Gao C: TRAF-interacting protein (TRIP) negatively regulates IFN- $\beta$ production and antiviral response by promoting proteasomal degradation of TANK-binding kinase 1. J Exp Med 209: 1703-1711, 2012

38. Chiang $\mathrm{C}$ and Gack MU: Post-translational control of intracellular pathogen sensing pathways. Trends Immunol 38: 39-52, 2017.

39. Chen C, Weisz OA, Stolz DB, Watkins SC and Montelaro RC: Differential effects of actin cytoskeleton dynamics on equine infectious anemia virus particle production. J Virol 78: 882-891, 2004.

40. Sodeik B: Mechanisms of viral transport in the cytoplasm. Trends Microbiol 8: 465-472, 2000.

41. Bozym RA, Delorme-Axford E, Harris K, Morosky S, Ikizler M, Dermody TS, Sarkar SN and Coyne CB: Focal adhesion kinase is a component of antiviral RIG-I-like receptor signaling. Cell Host Microbe 11: 153-166, 2012

42. Chiang HS, Zhao Y, Song JH, Liu S, Wang N, Terhorst C, Sharpe AH, Basavappa M, Jeffrey KL and Reinecker HC: GEF-H1 controls microtubule-dependent sensing of nucleic acids for antiviral host defenses. Nat Immunol 15: 63-71, 2014.

43. Ohman T, Rintahaka J, Kalkkinen N, Matikainen S and Nyman TA: Actin and RIG-I/MAVS signaling components translocate to mitochondria upon influenza A virus infection of human primary macrophages. J Immunol 182: 5682-5692, 2009. 